\title{
An Unusual Occurrence of Trifluoperazine-Induced Nystagmus in Persistent Hallucinatory Disorder- A Case Report
}

\author{
Avin Muthuramalingam¹ \\ ${ }^{1}$ Department of Psychiatry, Jawaharlal Institute of Postgraduate \\ Medical Education and Research, Karaikal, Puducherry, India \\ ${ }^{2}$ Department of Psychiatry, Mahatma Gandhi Medical College and \\ Research Institute (Deemed-to-be) University, Puducherry, India
}

Karthick Subramanian²

J Neurosci Rural Pract 2020;11:661-662

\begin{abstract}
Address for correspondence Vigneshvar Chandrasekaran, MD, Mahatma Gandhi Medical College and Research Institute (Deemed-to-be) University, Puducherry 607402, India (e-mail: vigneshvarchandrasekaran@gmail.com).
\end{abstract}
Abstract
Keywords
- adverse event reaction
- antipsychotic
- hypokalemia
- nystagmus
- trifluoperazine

Trifluoperazine is a conventional antipsychotic whose use has been limited with the arrival of relatively new atypical antipsychotics. However, conventional antipsychotics are utilized in the management of psychiatric illnesses comorbid with metabolic disorders such as diabetes or dyslipidemia. Though trifluoperazine has been known to cause extrapyramidal symptoms, rarely ophthalmic symptoms manifest. Here, we discuss the rare occurrence of newly-emergent nystagmus in an individual with persistent hallucinatory disorder and comorbid diabetes mellitus treated with trifluoperazine.

\section{Introduction}

Trifluoperazine was developed in the late 1950s and was utilized as a conventional antipsychotic in the treatment of psychotic disorders. Trifluoperazine, apart from dopamine blockade, exhibits anticholinergic and antiadrenergic properties leading to anxiolytic effects of the drug. ${ }^{1}$

Apart from the action on neuronal receptors, trifluoperazine also acts as a calmodulin modulator and an inhibitor of P-glycoprotein. ${ }^{2}$ Due to its effects on calmodulin receptors, trifluoperazine has been associated with cardiac adverse effects such as tachycardia, arrhythmia, and QT prolongation. ${ }^{1}$ Apart from cardiac side effects, trifluoperazine has been reported to cause cholestatic jaundice and electrolyte abnormalities such as hypokalemia. ${ }^{3,4}$

Psychotropics can lead to ocular adverse events among various other systemic side effects. Nystagmus, as an ocular adverse event, has been noted with mood stabilizers (lithium, carbamazepine, lamotrigine, topiramate) and benzodiazepines, but not with antipsychotics. ${ }^{5}$ We report a case of new-onset nystagmus following the administration of trifluoperazine in a patient with chronic psychosis.

\section{Case Report}

Mrs. A, a 53-year-old female, presented with auditory hallucinations, sleep disturbances, and irritability for a duration of 4 months. Upon clarification, the patient had a history of similar symptoms 5 years ago, which had resolved in a month by taking psychotropics on an outpatient basis. The treatment details were not available, though the patient remained off medications until the current presentation. Her medical history revealed type- 2 diabetes mellitus for the past 3 years and on regular oral hypoglycemic agents. There was no history of substance use in the patient. Family history was nil contributory. She was diagnosed with persistent hallucinatory disorder under "other non-organic psychotic disorders (F28)" group as per International Classification of Diseases, 10th revision Diagnostic Criteria for Research. ${ }^{6}$

The patient was started on risperidone ( $2 \mathrm{mg} /$ day) along with trihexyphenidyl (2 mg/day) initially. Risperidone dose was optimized to $4 \mathrm{mg} /$ day over the week based on symptom response. However, the response was inadequate and the patient reported excess sedation with risperidone $4 \mathrm{mg} /$ day. Hence, risperidone was cross-tapered 
with trifluoperazine in view of fewer side effects profile. Risperidone was gradually tapered (at the rate of $1 \mathrm{mg}$ every 3 days) and trifluoperazine was given at $2.5 \mathrm{mg} /$ day for the first week, which was later increased to $5 \mathrm{mg} /$ day. After 2 weeks, improvement in psychotic symptoms was noted with trifluoperazine $5 \mathrm{mg}$ /day that was evident by a $20 \%$ reduction from baseline in the scores of Brief Psychiatric Rating Scale (BPRS).

During the third week of trifluoperazine administration, the patient reported visual disturbances in the form of reduced vision leading to difficulty in walking. Clinical examination revealed newly emergent bilateral horizontal nystagmus. The nystagmus was binocular, conjugate, continuous, horizontal, right-beating with smooth pursuit ocular movements compensated by interspersed saccades. The nystagmus was not associated with change of head postures ruling out vestibular causes. There were no accompanying clinical features such as dysdiadochokinesia, intentional tremors, or past-pointing ruling out acute cerebellar pathology. No features of drug-induced parkinsonism or oculogyric crisis were noted. No clinical features suggestive of or seizures could be made out. The ophthalmological evaluation was found to be normal except for evident bilateral horizontal nystagmus and associated blurring of vision. The blood biochemistry returned normal results. The magnetic resonance imaging of the brain returned normal, which ruled out common causes of nystagmus (cerebellar atrophy, brainstem or midbrain lesions, multiple sclerosis). The clinical condition suggested a drug-induced cause for acute nystagmus due to trifluoperazine, which was immediately stopped. The horizontal nystagmus subsided within 48 hours of stopping the drug. The Naranjo scale for causality revealed a score of 7 implicating the nystagmus to be a "probable" adverse event due to trifluoperazine.

After 3 days, the patient was started on aripiprazole ( $5 \mathrm{mg} /$ day) in view of its lesser propensity to cause neurological side effects. Aripiprazole was optimized up to $15 \mathrm{mg} /$ day based on the clinical response. The patient had shown much improvement in psychotic symptoms affirmed by a $50 \%$ reduction in BPRS scores and had been on regular monthly follow-up for the past 3 months. Written informed consent was obtained from the patient prior to the write-up of the report.

\section{Discussion}

The above case scenario reports the occurrence of an acute onset of "bilateral horizontal nystagmus" as a neurological adverse event secondary to initiation of an antipsychotic (trifluoperazine). Naranjo scale for causality suggests nystagmus as a "probable" adverse drug event with trifluoperazine.?

The present report is one of the first reports of trifluoperazine-induced nystagmus. Trifluoperazine belongs to the class of phenothiazines and has lesser sedative and metabolic side effects favoring its use in the current patient profile.
Trifluoperazine has been previously reported to cause retinal pigmentary changes and accommodative deficits as ophthalmological side effects that were unobserved in the current report. ${ }^{5,8}$

Nystagmus has been reported in the presence of severe hypokalemia, which in turn has been reported in patients on therapeutic doses of trifluoperazine.,99 The reported patient was found to have normal levels of potassium and other electrolytes measured both at baseline and after the onset of nystagmus, ruling out hypokalemia as a possible mechanism of nystagmus. However, a transient change in potassium levels during the change of antipsychotics could not be ruled out in the current scenario.

We conclude by reporting the rare adverse effect of nystagmus with trifluoperazine at therapeutic doses. The evaluation of newly emergent nystagmus should also encompass the pharmacological profile of the patient along with the commonly considered metabolic and central nervous system causes. A keen outlook is warranted for prompt recognition of the rare adverse effects during switching and dose titration of psychotropics.

\section{Note}

The authors declare that the study was performed conforming to the Declaration of Helsinki.

\section{Authors' Contributions}

All the three authors contributed equally in case recruitment and manuscript preparation and the manuscript had been finalized and approved by all the three authors.

Funding

None.

\section{Conflict of Interest}

None declared.

\section{References}

1 Qin J,Zima AV, Porta M, Blatter LA, Fill M. Trifluoperazine: a ryanodine receptor agonist. Pflugers Arch 2009;458(4):643-651

2 Howland RH. Trifluoperazine: a sprightly old drug. J Psychosoc Nurs Ment Health Serv 2016;54(1):20-22

3 Rehman S, Chetia DJ. Hypokalemia with trifluoperazine at therapeutic dose. Indian J Psychol Med 2017;39(3):352-353

4 Sharifi V,AminiS, Ghaeli P, Saeedinia A. Trifluoperazine-induced cholestatic jaundice. Iran J Psychiatry 2010;5(3):117-118

5 Richa S, Yazbek J-C. Ocular adverse effects of common psychotropic agents: a review. CNS Drugs 2010;24(6):501-526

6 The ICD-10 classification of mental and behavioural disorders: diagnostic criteria for research. Available at: https://apps.who. int/iris/handle/10665/37108. Accessed December 19, 2019

7 Naranjo CA, Busto U, Sellers EM, et al. A method for estimating the probability of adverse drug reactions. Clin Pharmacol Ther 1981;30(2):239-245

8 Pula JH, Kao AM, Kattah JC. Neuro-ophthalmologic side-effects of systemic medications. Curr Opin Ophthalmol 2013; 24(6):540-549

9 Prasad A, Ghodsizad A, Pae W, et al. Non-cardiac symptoms of moderate to severe hypokalemia in a patient with a Syncardia ${ }^{\mathrm{TM}}$ total artificial heart. Heart Surg Forum 2016;19(1):E12-E13 